\title{
Neutrophil to Lymphocyte ratio of Synthetic Cannabinoid Intoxication
}

\author{
GOKHAN INANGIL, SULEYMAN DENIZ, FUAT GURBUZ, BARIS GUVEN, OMER BAKAL, MUSTAFA ZULKADIR, HUSEYIN SEN, SEZAI OZKAN \\ Department of Anesthesiology, Gulhane Military Medical Academy, Haydarpasa Training Hospital, Istanbul, Turkey
}

\author{
Corresponding author: \\ Gökhan İnangil \\ Department of Anesthesiology \\ Gulhane Military Medical Academy \\ Haydarpasa Training Hospital \\ Istanbul, Turkey
}

Phone: 00-90-532-7739003

Fax: 00-90-216- 3487880

E-mail: ginangil@gata.edu.tr

\section{ABSTRACT}

Synthetic cannabinoids represent an increasingly popular trend, and acute intoxication is widely seen in emergency rooms and intensive care units (ICU), as they are inexpensive and easily accessible. Cannabinoids mediate their effects through binding specific receptors which are members of the $G$ protein coupled receptor superfamily. Cannabinoid-2 receptors are primarily found in the immune system and mediate immunosuppression by inducing apoptosis, inhibition of proliferation and suppression of cytokine and chemokine production. Many studies have discussed the effects of cannabinoids on the hematological and immune systems but controversial results have been reported. The aim of this study was to identify laboratory findings of acute synthetic cannabinoid intoxication. Forty-two patients, admitted to the 'Anesthesia intensive care unit' between 2014 and 2015 with synthetic cannabinoid intoxication, were studied retrospectively to assess the relationship between synthetic cannabinoid intoxication and complete blood count (white blood cells (WBC), neutrophils, lymphocytes, monocytes, eosinophils, basophils) and neutrophil to lymphocyte ratio. WBC neutrophil count decreased at ICU discharge when compared to ICU admission $(\mathrm{p}<0.001)$. The ratio of neutrophils to lymphocytes was also lower at ICU discharge when compared to ICU admission $(\mathrm{p}<0.05)$.

Key words: synthetic cannabinoids, intoxication, neutrophil to lymphocyte ratio

\section{INTRODUCTION}

Synthetic cannabinoids represent an increasingly popular trend, and acute intoxication is widely seen in emergency rooms (ER) and intensive care units (ICU), as they are inexpensive and easily accessible. Use of synthetic cannabinoids has psychiatric, medical, and social consequences. They are found in powder form or loose leaf form. Powder form may be dissolved and sprayed onto a dried herbal substrate and packed, or purchased as a pre-rolled product. Various flavors may be added and they may contain different synthetic cannabinoids as well as preservatives, additives, amides, esters and benzodiazepines. These products are sold on the streets and they are typically smoked in cigarette papers or cannabis pipes. (1) Some of these products are marketed under different names and forms from head shops (a head shop is a retail outlet specializing in equipment used for consumption of cannabis and tobacco and items related to cannabis culture and related countercultures; products may include magazines (e.g., about cannabis culture, cannabis cultivation, tattooing and music) and gas stations). Ingredients change over time to avoid legislation. When a compound is banned, it is replaced by a pharmacologically similar compound to avoid regulations. (2) Thus, it can cause different clinical findings, depending on the ingredients.

Phytocannabinoids, or plant-derived cannabinoids, include delta-9-tetrahydrocannabinol (THC), the primary psychoactive component of cannabis. Cannabinoids mediate their effects through binding spe- cific receptors which are members of the $G$ protein coupled receptor superfamily. To date, two cannabinoid receptors have been identified: Cannabinoid-1 receptors (CB1) and Cannabinoid-2 receptors (CB2). CB1 are expressed primarily in the central nervous system (CNS) and are responsible for the psychoactive effects of cannabinoids by modulating neurotransmitter release. In contrast, $\mathrm{CB} 2$ are localized primarily in immune cells such as lymphocytes, macrophages and neutrophils and are responsible for immunomodulatory effects of cannabinoids. $(1,3,4)$ While THC is a low-efficacy partial agonist of cannabinoid receptors, the synthetic cannabinoids are high-potency full agonists for CB1 receptors. (5)

Most synthetic cannabinoids are not currently found using routine toxicology screening as they contain synthetic cannabinoids from different chemical classes. Some synthetic cannabinoids can be measured in the serum using chromatographic methods with mass spectrometry but these tests cannot be performed in many hospitals and they are time consuming and expensive. $(1,6)$ Single use of THC can be detected up to 3 days while daily use can be detected up to 10 days. (6)

While many of the acute effects are similar to the effects of cannabis, these effects may differ clinically in terms of intensity and it is unclear whether they are related to the differences between synthetic cannabinoids and THC or to the noncannabinoid components. (1) These effects include mood disorders (euphoria, negative mood), anxiety, psychosis, cognitive and 
neurological function disorders (dizziness, seizures, tremor, ataxia). Cardiovascular effects include tachycardia, bradycardia, ECG changes, and arrhythmias. Synthetic cannabinoids also induce dry mouth, reddened conjunctiva, pupillary changes including miosis and mydriasis, blurry vision and light sensitivity. In addition to psychiatric and physical symptoms, a number of acute changes in laboratory parameters have been reported in individuals who have intoxication. Hyperglycemia, hypokalemia, elevated creatinine, acidosis, elevated phosphokinase and elevated white blood cell count are reported. $(1,7)$

Many recent studies have indicated that the neutrophil to lymphocyte ratio (NLR) is a significant inflammatory marker in diagnosis of various diseases such as pulmonary thromboembolism, (8) malignancy, (9) cardiovascular (10) and inflammatory diseases. (11) It is also an inexpensive inflammatory marker that provides quick results. In this study we aimed to identify laboratory findings of acute synthetic cannabinoid intoxication and to assess the relationship between synthetic cannabinoid intoxication and NLR.

\section{MATERIAL AND METHODS}

A total of 56 patients admitted to the ICU between 2014 and 2015 with synthetic cannabinoid intoxication were studied retrospectively after approval by the local ethics committee. Synthetic cannabinoid intoxication information was obtained by witnesses or evidence such as loose leaf forms or rolled cigarettes found with the patient. All patients included in the study had used synthetic cannabinoids with inhalation and smoking, no ingestion case was reported. Forty-two patients, with laboratory results from admission to ER and discharge from ICU, were included in our study, whereas patients with missing data and with concomitant alcohol use (with blood ethanol levels more than $50 \mathrm{mg} /$ dL) were excluded. Laboratory results of complete blood count (WBC, neutrophils, lymphocytes, monocytes, eosinophils, and basophils) duration of ICU stay, age, and intubation data were recorded. Neutrophil Lymphocyte Ratio was calculated both on admission and discharge. Leukocytosis was defined as a WBC $>10.5 \times 103$ cells $/ \mu \mathrm{L}$ and leukopenia was defined as WBC count $<4.0 \times 103$ cells $/ \mu \mathrm{L}$. Received data was analyzed with SPSS 15.0. Continuous variables were represented as mean \pm SD. Ap- propriateness of the variables to a normal distribution was analyzed with Kolmogorov-Smirnov test. Data were compared with paired T Test. A $\mathrm{p}<0.05$ was deemed statistically significant.

\section{RESULTS}

A total of 42 patients were included in the study. All patients were male and aged between 21-28 (22 \pm 1.6$)($ mean \pm SD) years of age. Mean ICU duration was $1.66 \pm 0.4$ days. All patients had neurocognitive disorders, 4 patients (9.5\%) had respiratory disorders that needed intubation and mechanical ventilation. Laboratory tests at both admission and discharge were compared statistically. 24 patients $(57 \%)$ had leukocytosis and none of them had leukopenia on ER admission, and only 7 (16\%) patients had leukocytosis on discharge. WBC and neutrophil count decreased at ICU discharge when compared to ICU admission $(\mathrm{p}<0.001)$ (table 1$)$. NLR was found to be $5.5 \pm 4.7$ on admission and 2.7 \pm 2.2 on ICU discharge (table 1 ). The NLR was also decreased on ICU discharge when compared to ICU admission $(\mathrm{p}<0.05)$ (table 1).

Table 1. Comparison of data between intensive care unit (ICU) admission and discharge.

\begin{tabular}{llll}
\hline & ICU admission & ICU discharge & p \\
\hline WBC & $11.7 \pm 4.0$ & $8.7 \pm 2.7$ & $<0.001$ \\
\hline Neutrophil & $8.7 \pm 4.0$ & $5.5 \pm 2.8$ & $<0.001$ \\
\hline Lymphocyte & $2.11 \pm 1.01$ & $2.35 \pm 0.57$ & 0.161 \\
\hline Monocyte & $0.71 \pm 0.40$ & $0.65 \pm 0.26$ & 0.305 \\
\hline Eosinophil & $0.11 \pm 0.11$ & $0.15 \pm 0.11$ & 0.110 \\
\hline Basophil & $0.04 \pm 0.03$ & $0.03 \pm 0.04$ & 0.782 \\
\hline Platelet & $229.7 \pm 73.5$ & $215.7 \pm 53.3$ & 0.94 \\
\hline NLR & $5.5 \pm 4.7$ & $2.7 \pm 2.2$ & 0.01 \\
\hline
\end{tabular}

NLR, Neutrophil to lymphocyte ratio; WBC, White blood cells.

\section{DISCUSSION}

Cannabinoids are a group of compounds that mediate their effects through cannabinoid receptors $\mathrm{CB} 1$ and $\mathrm{CB} 2$, which are $\mathrm{G}$ protein coupled receptors found on immune system cells. While CB1 is expressed in the CNS and plays a role in modulating GABA and glutamate neurotransmission, $\mathrm{CB} 2$ is primarily found in the immune system and mediates immunosuppression by inducing apoptosis, inhibition of proliferation and suppression of cytokine and chemokine production. (12-14) THC is the major active ingredient in marijuana which is a low efficiency partial agonist of cannabinoid receptors. Synthetic cannabinoids are high efficiency full agonists of $\mathrm{CB}$ thus consequences of synthetic cannabinoid use differs from marijuana-like effects. Also some synthetic cannabinoids have long half-lives as a result of active metabolites. $(1,14)$

The neutrophil to lymphocyte ratio has been widely used in identifying the degree of inflammation, as white blood cell count and its subtypes play an important role in inflammatory processes. NLR is claimed to be more useful as it is a combination 
of two independent inflammatory markers. $(15,16)$ However, no studies have been found regarding NLR in acute synthetic cannabinoid intoxication.

Many studies have discussed the effects of cannabinoids on the hematological and immune system but controversial results have been reported. Oseni at al. studied the effects of marijuana smoking on some hematological parameters, including WBC, hemoglobin, platelets and NLR, and found no significant difference with non-smokers in contrast to our study. (16) However they included smokers that used marijuana for at least two years which differs from our study population, as inexperienced smokers had symptoms of dizziness, tachycardia, tremor and disorientation while experienced users did not.

Altınısık et al. reported a series of synthetic cannabinoid intoxication cases, followed up in ICU, with leukocytosis in 33\%, and leukopenia in $8 \%$ of cases. (7) However they mainly focused on clinical presentation of intoxication. In our study 24 pa- tients (57\%) had leukocytosis and none of them had leukopenia on ER admission, and only 7 (16\%) patients had leukocytosis on discharge.

Mukhtar et al. reported a decrease in total WBC count and neutrophil count in rats treated with intramuscular cannabinoid extract administration compared to the control group. Another animal study by Obembe et al. reported a decrease in total WBC in groups fed different doses of cannabis sativa solution. Authors attributed the results to bone narrow supression which disturbs maturation of monocytes, as the design of these studies aimed at effects of chronic use. $(17,18)$

Limitations of our study include the relatively small sample size and our retrospective study design. Also we could not determine or obtain any information about the type of synthetic cannabinoid used, which may have affected our results. We also did not know if the patient was a chronic user of synthetic cannabinoids, other drugs or cannabis, which may have affected our re- sults. As we focused on acute intoxication of synthetic cannabinoids, we compared WBC and NLR of patients admitted to ICU with an altered level of consciousness, with their control blood samples taken at discharge. Our study would be more valuable if we had obtained follow up results over a larger time interval.

\section{CONCLUSIONS}

Neutrophil count and neutrophil to lymphocyte ratio were decreased in discharge blood samples indicating hematological characteristics of synthetic cannabinoid intoxication. Cannabinoids have a potential use as anti-inflammatory agents against inflammatory and autoimmune diseases as well as for, treating sepsis. However synthetic cannabinoids differ from cannabinoids with their high potency and full agonist properties and therefore further studies should be performed on this subject.

\section{REFERENCES}

1. Spaderna M, Addy PH, D’Souza DC. Spicing things up: synthetic cannabinoids. Psychopharmacology 2013;228:525-40.

2. Dargan PI, Hudson S, Ramsey J, Wood DM. The impact of changes in UK classification of the synthetic cannabinoid receptor agonists in 'Spice'. Int J Drug Policy 2011;22:274-7.

3. Lehmann C, Kianian M, Zhou J, Cerny V, Kelly M. The endocannabinoid system in sepsis - a potential target to improve microcirculation? Signa Vitae 2011;6:7-13.

4. Sardinha J, Lehmann C, Kelly M. Targeting the Endocannabinoid System to Treat Sepsis. Signa Vitae 2013;8:9-14.

5. Atwood BK, Lee D, Straiker A, Widlanski TS, Mackie K. CP47,497-C8 and JWH073, commonly found in 'Spice' herbal blends, are potent and efficacious CB(1) cannabinoid receptor agonists. Eur J Pharmacol 2011;659:139-45.

6. Ščavničar A, Granić P, Lovrić M, Rogić D. Toxicological screening - Experience from University Hospital Centre Zagreb Emergency Medical Service. Signa Vitae 2015;10(SUPPL 1):92-6.

7. Altınışık U, Altınışık HB, Şimşek T, Doğu T, Şimşek E, Aydın H. Clinical Characteristics of Patients Diagnosed with Synthetic Cannabinoid (Bonsai) Intoxication in ICU. J Turk Soc Intens Care 2015;13:117-21.

8. Cavuş UY, Yildirim S, Sönmez E, Ertan C, Ozeke O. Prognostic value of neutrophil/lymphocyte ratio in patients with pulmonary embolism.Turk J Med Sci 2014;44:50-5.

9. Ozdemir Y, Akin ML, Sucullu I, Balta AZ, Yucel E. Pretreatment neutrophil/lymphocyte ratio as a prognostic aid in colorectal cancer. Asian Pac J Cancer Prev 2014;15:2647-50.

10. Arbel Y, Finkelstein A, Halkin A, Birati EY, Revivo M, Zuzut M, et al. Neutrophil/lymphocyte ratio is related to the severity of coronary artery disease and clinical outcome in patients undergoing angiography. Atherosclerosis 2012;225:456-60.

11. Gao SQ, Huang LD, Dai RJ, Chen DD, Hu WJ, Shan YF. Neutrophil-lymphocyte ratio: a controversial marker in predicting Crohn's disease severity. Int J Clin Exp Pathol 2015;8:14779-85.

12. Rieder SA, Chauhan A, Singh U, Nagarkatti M, Nagarkatti P. Cannabinoid-induced apoptosis in immune cells as a pathway to immunosuppression. Immunobiology 2010;215:598-605.

13. Nagarkatti P, Pandey R, Rieder SA, Hegde VL, Nagarkatti M. Cannabinoids as novel anti-inflammatory drugs. Future Med Chem 2009;1:1333-49.

14. Seely KA, Lapoint J, Moran JH, Fattore L. Spice drugs are more than harmless herbal blends: a review of the pharmacology and toxicology of synthetic cannabinoids. Prog Neuropsychopharmacol Biol Psychiatry 2012;39:234-43.

15. Polat O, Yavaş GF, İnan S, İnan ÜÜ. Neutrophil-to-Lymphocyte Ratio as a Marker in Patients with Non-arteritic Anterior Ischemic Optic Neuropathy. Balkan Med J 2015;32:382-7.

16. Oseni BS, Togun VA, Taiwo OF. Effect of Marijuana Smoking on Some Hematological Parameters of Smokers. World J Med Sci 
2006;1:82-5.

17. Mukhtar AH, Elbagir NM. Effect of Cannabis sativa on Hematological Indices in Rats and Men. Pak J Nutr 2011;10:313-16.

18. Obembe, AO, Omini GC, Okon UA, Okpo-ene AI, Ikpi DE. Hematological and Immunological Effect of Cannabis sativa on Albino Wistar Rats. BJMMR 2015;7:52-60. 